\title{
SPATIAL DATA MINING ON REMOTE SENSING PERSPECTIVE
}

\author{
Josephina Paul ${ }^{1}$
}

Abstract: Spatial data mining has been evolved as a special branch of data mining since the last two decades as the conventional data mining algorithms fail to produce good results from large spatial databases. Satellite images carry rich information of the geographical coverage area, which can be harnessed using efficient algorithms that consider spatial aspects into, while processing. Several algorithms, that are build up on the state-of-the-art techniques such as AI, ANN, SVM, Fuzzy logic, and are categorized into the classical grouping of clustering, classification and frequent pattern mining have grown dimensionally and have stretched into multidisciplinary research areas. In this letter, we will discuss the various efforts taken in the last two decades in the field of spatial data mining on the perspective of satellite/ remote sensing images.

Keywords: Knowledge discovery, Remote sensing, Satellite images, Spatial data mining

\section{INTRODUCTION}

It has been seen that a special interest in the spatial data mining for the past two decades as the technology to collect the aerial images of even remote locations have grown affluently. An abundant store of remote sensing /satellite data is available with the major space research agencies of various nations spread across the continents. Satellite images carry rich spatial information of the coverage area, which includes land use, land cover, vegetation, crops and cultivation, regional population, water bodies, soil, unutilized cultivars, buildings, roads and everything on the surface of the geographical area. The images procured at different time stamps can tell stories of the life systems and its transformations on various perspectives. Therefore, the spatial, temporal and spatial-temporal data mining of satellite images has been emerged as an important branch in data mining research. Similarly, it has become an enthusiastic research area for the researchers of diverse disciplines as it help in decision making directly or indirectly.

As these images contain multispectral bands and incorporate several features, they occupy disk spaces of size in the gigabyte terms or more, the processing of images in time bound manner has become a real challenge for the scientists. The usual techniques of classification, clustering and association rule mining for the frequent patterns under the spatial paraphrase can made available to detect and discover unusual patterns and relationships of the objects that are encapsulated in

${ }^{1}$ KCAET, Tavanur 
the images. In this review, we will discuss the various efforts taken in the last two decades in the field of spatial data mining in the perspective of satellite/ remote sensing images.

Knowledge discovery in databases (KDD) or data mining is the non-trivial extraction of implicit, previously unknown, and potentially useful information from databases [FPM 1991]. So the 'mined' information is typically represented as a model of the semantic structure of the dataset, where the model may be used on new data for prediction or classification. Spatial data mining and knowledge discovery (SDMKD) is the process of discovering interesting and previously unknown, but potentially useful patterns from spatial databases (2008). Types of the knowledge that discovered from spatial database usually include spatial characterization, spatial classification, spatial dependency, spatial association, spatial clustering and spatial trend/outlier analysis. Spatial co-location pattern mining is spiritually similar to, but technically very different from, association rule mining. Co-location patterns represent subsets of features frequently located together (2009, p.403-408).

Temporal data mining is concerned with data mining of large sequential data sets. By sequential data, we mean data that is ordered with respect to some index. For example, time series constitute a popular class of sequential data, where records are indexed by time (Jiawei, 2004). Spatiotemporal data mining presents a number of challenges due to the complexity of geographic domains, the mapping of all data values into a spatial and temporal framework, and the spatial and temporal autocorrelation exhibited in most spatio-temporal data sets (Miller et al., 2001).

Before detailing the specific works done in the presented topic, a peep into some of the available state-of-the-art data mining techniques would be relevant.

\section{DATA MINING TASKS}

DM tasks can be broadly classified into two categories: descriptive and predictive. While the general properties of the data is being characterized in the descriptive tasks, the delineating predictions on the on the current data is performed in predictive tasks. There are many and diverse data mining tasks such as classification, regression, association analysis, clustering, time series analysis and outlier analysis are found in the literature

\subsection{Classification}

Data classification is a two-step process consisting of a learning step or training phase and a classification step, where the model is used to predict class labels for the given data. Because the class label of each training tuple is provided, this step is also known as supervised learning (Jiawei, 2004). The maximization of the classification accuracy rate in the test set is the main goal of learning. Prediction is similar to classification, but the difference is that the goal or predicted attribute is continuous valued or ordered rather than discrete valued class labels as in classification. 
The important classification methods are decision tree induction, Bayesian classification method, rule based classification and neural network based classification, support vector machines, fuzzy sets and model based approaches.

\subsection{Clustering}

Clustering is an unsupervised learning task where one seeks to identify a finite set of categories termed clusters to describe the data (Everitt, 1993, Kaufman, 1990). Unlike classification that analyses class-labeled instances, clustering has no training stage, and is usually used when the classes are not known in advance. The grouping of data into clusters is based on the principle of maximizing the intra-class similarity and minimizing the interclass similarity. The clustering algorithms can be grouped into four classes: partitioning algorithms, hierarchical algorithms, density-based algorithms and grid-based algorithms.

The partition based algorithms divide the dataset into $\mathrm{k}$ partitions in such a way that each object belongs to at most one partition. The oldest algorithm K-means and K-medoids- a variation of Kmeans, cluster the data around the centroid of the cluster based on the Euclidean distance. CLARA (Clustering Large Applications) and CLARANS (Clustering Large Applications) are the algorithms developed for very large datasets. In hierarchical method, a hierarchical decomposition of data is done and represented as a dendrogram. A hierarchical method can be either agglomerative which uses a bottom up (merging) strategy or divisive that uses top down (splitting) strategy Hierarchical algorithms do not need $\mathrm{k}$ as an input parameter, which is an obvious advantage over partitioning algorithms. The popular hierarchical algorithms are BIRCH (Balanced Iterative Reducing and Clustering Using Hierarchies), CURE (Clustering Using REpresentatives) and CHAMELION, in all these, spatial attribute has not been taken into account. The key idea in density based method is that, for each point of a cluster, the neighborhood of a given radius has to contain at least a minimum number of points, i.e., the density in the neighborhood has to exceed some threshold. They can discover clusters of arbitrary shape. DBSCAN (Density-Based Spatial Clustering of Applications with Noise), OPTICS (Ordering Points To Identify the Clustering Structure), DENCLUE (DENsity-based CLUStEring) and DBCLASD (Distribution Based Clustering of LArge Spatial Databases) are some of density based algorithms. Grid based algorithms quantize the space into a finite number of cells and then do all operations on the quantized space. The main characteristic of these approaches is their fast processing time, which is typically independent of the number of data objects. They depend only on the number of cells in each dimension in the quantized space. STING (STatistical INformation Grid) and WaveCluster algorithms are working on the principle of clustering on grids. While all the above methods create crisp clusters fuzzy clustering approach creates clusters based on membership values of clusters on data and therefore, a data object can falls in more than one cluster. Fuzzy C-Means algorithm is one of the oldest fuzzy clustering algorithms. Many variations of fuzzy C-Means have been in use since its invention by Bezdek in 1981.

\subsection{Association and Mining Frequent Patterns}

Frequent patterns are patterns that appear in a data set frequently. A set of items such as milk and bread that appear together is called frequent item set (Jiawei, 2004). The frequent pattern can be represented in terms of association rules. Rule support and confidence are two measures of rule 
interestingness. They reflect respectively the usefulness and certainty of the discovered rules. Typically, association rules are considered interesting if they satisfy both a minimum support threshold and a minimum confidence threshold.

\section{SPECIFIC STUDIES ON SPATIAL DATA MINING}

While scanning the literature, we could find a handful of novel algorithms in the group of spatial knowledge discovery which was developed for variety of applications. A brief description on that is detailed.

Wadii Boulila et al. (2010) introduced a new spatio-temporal technique for the knowledge discovery from the satellite images. The key step in their study is the multi-approach image segmentation. In order to reduce the loss of information while segmenting the images, they applied simultaneously, the five segmentation methods available. They used the k-means algorithm as a partition-based method, the Balanced Iterative Reducing and Clustering (BIRCH) algorithm as a hierarchical method, STatistical INformation Grid (STING) algorithm as a gridbased segmentation method, Density Based Spatial Clustering of Applications with Noise (DBSCAN) algorithm for the density based segmentation method and as a model-based segmentation method, the Expectation-Maximization (EM). They have also taken into consideration, the spatial and temporal components in the images. For the same, the multiapproach model mining, based on four steps : fuzzy clustering, similarity measure, fuzzy classification and spatio-temporal change tree building was applied. Cosine similarity was used for calculating the resemblance between two spatial-temporal models.

In order to explore the spatial and temporal relationships among a set of variables that characterize socioeconomic and land cover change in the Denver, Colorado, USA region from 1970-1990, association rule mining was used by Jeremy Mennis and Jun Wei Liu and they obtained promising results.

Deepti Joshi et al.(2012) have developed the Constrained Polygonal Spatial Clustering (CPSC) algorithm based on the A_search algorithm that integrates cluster-level and instance-level constraints as heuristic functions, for the redistricting of geographical area. Using these heuristics, CPSC identifies the initial seeds, determines the best cluster to grow, and selects the best polygon to be added to the best cluster.

A segmentation technique developed by Leen-Kiat Soh and Costas Tsatsoulis (1999) integrates traditional image processing algorithms with techniques adapted from data mining to analyze and segment unstructured satellite images of natural scenes.

Artificial Neural Net based algorithms are well proven methods in the field of classification based on training of data. Various kinds of networks ranging from multi layer perceptrons to self organizing feature maps have been developed for the spatial knowledge extraction from remote sensing images. Fabio Del Frate (2007) assessed the performance and effectiveness of multilayer perceptron (MLP) networks as a tool for the classification of remotely sensed images at very high or high resolution. Limin Jiao and Yaolin Liu [41] proposed a spatial clustering model based on self-organizing feature map and a composite distance measure, for the knowledge discovery from spatial database of spatial objects with non-spatial attributes. 
Fuzzy set based clustering can generate near realistic clusters than the hard clustering algorithms. While the conventional clustering algorithms allot the data into exactly one cluster, the fuzzy algorithms defines membership values to more than one cluster, for each data, as a pixel can include different classes of objects. S. Bandyopadhyay (2005, p.579-593) has devised a genetically guided fuzzy clustering algorithm that incorporates spatial information inherent in the pixels and could obtain good segmentation results. Two different approaches, WinAvg and WinTopDown, have been implemented for incorporating spatial information. As a result, the feature space is enhanced, so that its dimensionality increases from $\mathrm{N}$ (the number of bands in the image) to $2 \mathrm{~N}$ and $3 \mathrm{~N}$, respectively. Muhammad Shahbaz et al.(2012) has put forward a method to classify the satellite images by identifying objects in the imagery with respect to organization of color pixels and pixel intensity. Extracted attributes help to identify objects that lie inside the satellite image. Keh-Shih Chuang et al. (2006, p.9-15) presented a fuzzy c-means (FCM) algorithm that incorporates spatial information into the membership function for clustering.

Marcelino Pereira S. Silva et al. (2003) proposed a technique for the structural classification and pattern mining of satellite images using domain concepts to build generic description of patterns in remote sensing images, and to identify such patterns in images with the help of decision trees. Yuliya Tarabalka et al. (2009) proposed a spectral-spatial classification scheme for hyperspectral images that combines the results of a pixel wise support vector machine classification and the segmentation map obtained by partitional clustering using majority voting.

Mihai Datcu et al. (2003) have devised an algorithm based on Bayesian networks, for the knowledge driven content based image mining of remote sensing images. L.Gomez-Chova et al. (2003) proposed a dimensionality reduction strategy that eliminates redundant information, by means of local correlation criterion between contiguous spectral bands, and a subsequent selection of the most discriminative features based on a Sequential Float Feature Selection (SFFS) algorithm for the processing of hyper spectral satellite images. A Linear Regression ( LR) model was devised by Qi Cheng et al (2006) for both the feature selection and the classification of remotely sensed images, where more informative soft classifications are produced naturally.

In order to determine the land cover and change detection of diverse locations over the world, many studies have been conducted using satellite/remote sensed imageries applying a number of algorithms of data mining on it. A few novel algorithms have been developed in some of these studies.

Hongtau $\mathrm{Hu}$ and Yifang Ban (2008) classified the urban land-cover and detected the changes in land-cover over a period of 1988 to 2002 in the urban area of Beiging, China, using neural networks and rule based classifiers on Radarsat SAR data. Kathlein M. Bergen et al. (2005, p.431-446) successfully derived the land cover changes over a period from 1980-2000 of forests, urban and agricultural categories over a seven state region in US, from the AVHRR satellite imagery. They used the Multi Objective Land Allocation (MOLA) procedure and unsupervised classification using ISODATA tool in ISOCLUS program and could obtain $85 \%$ and $82 \%$ accuracy for the MOLA and ISODATA respectively. For detecting land cover change by 
analyzing the Landsat imagery over a period of 1986 and 2001, J.R. Otukei and T. Blaschke (2010) evaluated the potential of Decision Trees for data mining and they obtained better accuracies for DTs than SVMs, and MLC. In the experiments of mining the agricultural land use evolution from satellite images, Jean-Francois Mari and Florence Le Ber (2006, p.406-414) showed that the HMM2's are powerful tools for temporal and spatial data mining.

In order to investigate the temporal changes in the cultivated area, the satellite remote sensing data would prove to be the most effective data source (Delgado, 2009). Extensive research has been carried out to use various data mining techniques on remote sensing/satellite images for the crop classification and detection with the spatial perspective.

Early estimation of crop surfaces and agricultural monitoring (from May 1997 to January 1998), using the RADARSAT data of the test site of the West-Flevoland polder in the Netherlands was done by Francis Yakam et al. and they could discriminate between cultivated/non-cultivated surfaces and identified the major crop types. They used SAR time series decomposition and ISODATA for the analysis and calculated the crop acreages in various agricultural seasons: spring, summer etc. Omkar et al. (2008, p. 175-182) presented the crop classification of a cultivated region using various algorithms - Particle Swarm Optimisation (PSO) and Ant Colony Optimisation (ACO) Techniques and conventional statistical method, the Maximum Likelihood Classifier (MLC) on high resolution satellite image.

S.P. Vyas et al. (2005) have experimented with a multi-crop separability study using hierarchical decision rule based supervised classification and could identify a total of 24 classes comprising various land covers like wheat, mustard, potato, fallow, forest, sand, water body, urban etc. Using multi-date LISS-III data, it was possible to discriminate and map the various crops in the study area, whereas single study date is still good for homogeneous area and reduces the data volume.

Turgay Celik and Kai-Kuang Ma (2010) proposed an unsupervised change-detection method for multi-temporal satellite images. An approach that relies on frequent sequential pattern extraction along the temporal dimension, combined with a spatial connectivity criterion was presented by Andreea Julea (2012, p. 23-44). In order to assist agricultural men in decision making, Han Wen Hsiao et al (2006) proposed a 2-phase hierarchical clustering method to mine co-location patterns from geographical data that have applications in agriculture. Eg. To find the occurrence of insect's pest in grassland where the landscape, wind direction and wind velocity are strongly correlated in a particular way.

Some of the data mining techniques are related to soil mapping and modeling. Christensen and Di Cook (1998) describe simple numerical methods to establish the relationship between 10 soil characteristic variables and corn yield. Yang et al (1999, p.60-65) used remote sensing techniques in conjunction with AI neural networks to identify weeds in corn fields. Christopher J. Moran et.al (2002, p. 533- 549) modeled the soil map of Murray Basin, Australia using induction rules based on the spatial environmental data. Tree pruning and boosting were used to produce better classification results of soil data. A study was conducted in 2009 by P. Bhargavi and S. Jyoti on the classification of agricultural land soils using Naïve Bayes data mining 
technique. The results were $100 \%$ classified when Naive Bayes Classifier applied to the soil dataset.

\section{CONCLUSION}

The application and usage of satellite images are innumerous as it carries loads of information relevant to diversified fields. The geographers, town planners and remote sensing scientists, civil engineers, agriculture scientists and environmental researchers use the potential of spatial coupled with temporal features for formulating new findings and ideas that help in decision making directly or indirectly. Spatial data mining has become a useful tool for scientists and researchers of diverse disciplines as this can be used for knowledge discovery relevant to the field of their interest, which in turn, increases the demand of research to devise novel algorithms and techniques in spatial and temporal data mining. This relatively young research field has come up with valuable contribution in its short journey raises hope of bringing more outputs in its coming years.

\section{REFERENCES}

[1]. Andreea Julea et. al: 2012a, 'Efficient Spatio-temporal Mining of Satellite Image Time Series for Agricultural Monitoring', (Transactions on Machine Learning and Data Mining), 5(1), 23-44.

[2]. Andreea Julea; Nicolas M'eger; Christophe Rigotti; Emmanuel Trouv'e; Romain Jolivet; Philippe Bolon: 2012b, 'Efficient Spatio-temporal Mining of Satellite Image Time Series for Agricultural Monitoring', Transactions on Machine Learning and Data Mining 5,(1) 23-44. ibai-publishing, ISSN:1865-6781, ISBN: 978-3-942952-11-8.

[3]. Bhargavi, P. and Jyothi, S: 2009, 'Applying Naive Bayes Data Mining Technique for Classification of Agricultural Land Soils', (IJCSNS International Journal of Computer Science and Network Security), 9(8).

[4]. Christensen,W. F., and Di Cook: 1998, 'Data Mining Soil Characteristics Affecting Corn Yield', (citeseer.nj.nec.com/christensen98data.html).

[5]. Christopher, J; Moran and Elisabeth, N. B.: 2002, 'Spatial data mining for enhanced soil map modeling', (Int. j. geographical information science), 16(6), 533- 549.

[6]. Deepti Joshi; Leen-Kiat Soh and Ashok Samal: 2012, 'Redistricting Using Constrained Polygonal Clustering', (IEEE Transactions On Knowledge And Data Engineering), 24(11).

[7]. Diansheng Guo, a; Jeremy Mennis: 2009, 'Spatial data mining and geographic knowledge discovery-An introduction', (Computers, Environment and Urban Systems), 33, 403-408.

[8]. Delgado, G. et.al: 2009, 'Using fuzzy data mining to evaluate survey data from olive grove cultivation', (Computers and Electronics in Agriculture), 65(1), 99-113.

[9]. Ester, M; H-P Kriegel, Sander; X. Xu: 1996, 'A density-based algorithm for discovering clusters in large spatial databases with noise', (In Second International Conference on Knowledge Discovery and Data Mining), 226-231. AAAI Press.

[10]. Everitt, B. S.: 1993, 'Cluster Analysis', (Edward Arnold).

[11]. Francis Yakam-Simen, Edmond Nezry and Francis Zagolski: 'Early Estimation of Crop Surfaces, and Agriculture Monitoring using RADARSAT Data', (Internet Homepage: http://www.treemail.nl/privateers).

[12]. Fabio Del Frate; Fabio Pacifici; Giovanni Schiavon and Chiara Solimini: 2007, 'Use of Neural Networks for Automatic Classification From High-Resolution Images', (IEEE Transactions On Geoscience And Remote Sensing), 45(4).

[13]. Gomez-Chova, L: 2003, 'Feature selection of hyperspectral data through local correlation and SFFS for crop classification', (IEEE).

[14]. Han Wen Hasiao; Meng-shu Tsai and Shao-Ching Wung: 2006, ;Spatial Data Mining of Colocatin Patterns for Decision Support in Agriculture', (Asian Journal of Health and Information Sciences. http://www.seismo.ethz.ch/). 
[15]. Hongtao Hu and Yifang Ban: 2008, 'Urban Land-Cover Mapping and Change Detection with Radarsat Sar Data using Neural Network and Rule-Based Classifiers', (The International Archives of the Photogrammetry, Remote Sensing and Spatial Information Sciences, Beijing), XXXVII(B7).

[16]. Jean-Francois Mari Florence Le Ber: 2006, 'Temporal and Spatial Data Mining with Second-Order Hidden Markov Models', (Soft Computing), 10, 406-414.

[17]. Jiawei, Han and Micheline, Kamber: 2004, 'Data Mining Concepts and Techniques', second Edition, Elsevier

[18]. John, F. R, and Myra Spiliopoulou: 1999, 'A Bibliography of Temporal, Spatial and Spatio-Temporal Data Mining Research', (SIGKDD Explorations, ACM SIGKDD), 1(1), 34-38.

[19]. Kathleen, M, Bergen; Daniel, G, Brown; James, F. R; Eric, J. G: 2005, 'Change detection with heterogeneous data using ecoregional stratification,statistical summaries and a land allocation algorithm', (Remote Sensing of Environment), 97, 434-446.

[20]. Kaufman, L. and Rousseeuw, P.J: 1990, 'Finding Groups in Data: An Introduction to Cluster Analysis', (John Wiley \& Sons).

[21]. Keh-Shih Chuang et. al: 2006, 'Fuzzy c-means clustering with spatial information for image segmentation', (Computerized Medical Imaging and Graphics), 30, 9-15.

[22]. Leen-Kiat Soh, and Costas Tsatsoulis: 1999, 'Segmentation of Satellite Imagery of Natural Scenes Using Data Mining', (IEEE Transactions On Geoscience And Remote Sensing), 37(2).

[23]. Limin Jiao and Yaolin Liu: 2008, 'Knowledge discovery by spatial clustering based on self-organizing feature map and a composite distance measure', (The international Archives of the Photogrammetry, Remote Sensing and Spatial Information Sciences), XXXVII(B2), Beijing.

[24]. Marcelino Pereira, S. Silva; Gilberto Câmara; Ricardo Cartaxo M. Souza; Dalton M. Valeriano; Maria Isabel S. Escada: 'Mining Patterns of Change in Remote Sensing Image Databases'.

[25]. Mihai Datcu; Herbert Daschiel; Andrea Pelizzari; Marco Quartulli; Annalisa Galoppo; Andrea Colapicchioni; Marco Pastori; Klaus Seidel; Pier Giorgio Marchetti and Sergio D'Elia: 2003, 'Information Mining in Remote Sensing Image Archives: System Concepts', (IEEE Transactions On Geoscience And Remote Sensing), 41(12).

[26]. Miller, H. and Han, J: 2001, 'Geographic Data Mining and Knowledge Discover'. Taylor and Francis.

[27]. Muhammad Shahbaz; Aziz Guergachi; Aneela Noreen; Muhammad Shaheen: 2012, 'Classification by Object Recognition in Satellite Images by using Data Mining', (Proceedings of the World Congress on Engineering, London, U.K), 1.

[28]. Otukei, J.R; Blaschke, T: 2010, 'Land cover change assessment using decision trees, support vector machines and maximum likelihood classification algorithms', (International Journal of Applied Earth Observation and Geoinformation) 12.

[29]. Omkar, S. N et al: 2008, 'Crop Classifi cation using Biologically-inspired Techniques with High Resolution Satellite Image', (Journal of Indian Soc. Remote Sensing), 6(36), 175-182.

[30]. Qi Cheng; Pramod, K. V. and Manoj, K. A.: 2006, 'Logistic Regression for Feature Selection and Soft Classification of Remote Sensing Data', (IEEE Geoscience And Remote Sensing Letters), 3(4).

[31]. S. Bandyopadhyay : 2005, 'Satellite image classification using genetically guided fuzzy clustering with spatial information', (International Journal of Remote Sensing), 26(3), 579-593.

[32]. Shahin, M. A et.al: 2001. 'Artificial intelligence classifiers for sorting apples based on watercore', ( J. Agric. Eng. Res), 79(3), 265-274.

[33]. Shekhar, $\mathrm{S}$ et. al: 'Data Mining and Visualization of Twin-Cities Traffic Data, http://www.cs.umn.edu/Research/shashi-group'.

[34]. Sudhanshu Sekhar Panda; Daniel, P. A; Suranjan Panigrahi: 2010, 'Application of Vegetation Indices for Agricultural Crop Yield Prediction Using Neural Network Techniques', (Remote Sensing), 2, 673-696.

[35]. Turgay Celik and Kai-Kuang Ma: 2010, 'Unsupervised Change Detection for Satellite Images Using DualTree Complex Wavelet Transform', (IEEE Transactions On Geoscience And Remote Sensing), 48(3).

[36]. Vyas, S. P; Oza, M. P. and Dadhwal, V.K: 2005, 'Multi-Crop Separability Study Of Rabi Crops Using Multi-Temporal Satellite Data', (Journal of the Indian Society of Remote Sensing), 33(1). 
[37]. Wadii, Boulila; Imed Riadh Farah; Karim Saheb Ettabaa; Basel Solaiman; Henda Ben Ghézala: 2010, 'Spatio-Temporal Modeling for Knowledge Discovery in Satellite Image Databases'.

[38]. Xiangming Xiao, $\mathrm{T}$ et al.: 2005, 'Mapping paddy rice agriculture in southern China using multi-temporal MODIS images', (Remote Sensing Environment), 95, 480-492.

[39]. Yang, C.; Prasher, S. O. and Landry. J. A: 1999, 'Use of artificial neural networks to recognize weeds in a corn field. Journée d'information scientifique et technique en génie agroalimentaire', (Saint-Hyacinthe QC, Canada), 60-65.

[40]. Yuliya Tarabalka; Jón Atli Benediktsson; Jocelyn Chanussot: 2009, 'Spectral-Spatial Classification of Hyperspectral Imagery Based on Partitional Clustering Techniques', (IEEE Transactions On Geoscience And Remote Sensing), 47(8). 